\title{
Hypersingular integral equations for arbitrarily located planar cracks in an anisotropic elastic bimaterial
}

\author{
W.T. Ang* \& Y.S. Park \\ Faculty of Engineering, Universiti Malaysia Sarawak, 94300 Kota Samarahan, Malaysia
}

(Received 8 January 1997; accepted 29 May 1997)

\begin{abstract}
Hypersingular integral equations are derived for the problem of arbitrarily located planar cracks lying in the interior of two dissimilar anisotropic elastic half-spaces which adhere perfectly to each other. The unknown functions in the integral equations are the crack-opening displacements. The integral equations are solved numerically for specific examples involving particular transversely isotropic materials in order to compute physical quantities of interest such as the crack tip stress intensity factors or the crack energy. $\mathbb{C} 1997$ Elsevier Science Ltd.
\end{abstract}

Key words: Anisotropic bimaterial, hypersingular integral equations, planar cracks.

\section{INTRODUCTION}

During the last 10 years or so, there has been a growing interest among many researchers in formulating crack problems using hypersingular integral equations ${ }^{1-6}$. An advantage of using such an integral formulation is that the unknown functions in the integral equations are directly related to the crack-opening displacements. Furthermore, effective numerical methods for solving the integral equations, such as the collocation technique ${ }^{7}$, are available.

In the present paper, the plane problem of several arbitrarily located planar cracks in two dissimilar anisotropic elastic half-spaces which adhere perfectly to each other is formulated in terms of a system of hypersingular integral equations. The cracks are assumed to open up under the action of suitably prescribed internal tractions. In the formulation, the continuity conditions on the interface between the dissimilar materials are enforced exactly. Thus, in the numerical solution of the integral equations, discretization of the interface is conveniently avoided.

The integral equations derived are valid for the most general anisotropic materials. The materials are not required to possess any symmetries in their anisotropy. However, we solve those equations numerically using a collocation technique for only specific examples of the problem, involving

*To whom correspondence should be addressed. particular transversely isotropic materials. Numerical results for the crack tip stress intensity factors or the crack energy are obtained.

From a practical standpoint, the problem under consideration may be of useful relevance to composite and anisotropic structures which can now be found in an increasingly wider range of applications in modern technology. The need to assess the reliability and integrity of these structures has indeed generated much interest among many researchers in the analysis of stress in anisotropic layered materials, particularly those containing cracks ${ }^{5,8-13}$.

\section{STATEMENT OF THE PROBLEM}

Referring to an $0 x_{1} x_{2} x_{3}$ Cartesian coordinate system, consider an infinite elastic medium which comprises two regions: $x_{2}>0$ (region 1 ) and $x_{2}<0$ (region 2). The regions are occupied by two possibly different anisotropic materials which adhere rigidly to each other along the $x_{2}=0$ interface. There are several arbitrarily located planar cracks in the elastic medium. It is assumed that the geometries of the cracks do not vary along the $x_{3}$-direction and the cracks do not intersect with one another or the interface separating the two regions.

Let us denote those cracks in region 1 (if any) by $C^{(1)}$, $C^{(2)}, \ldots, C^{(N-1)}$ and $C^{(\mathrm{N})}$ and any other remaining cracks in 